\title{
Serum Levels and Adipose Tissue Gene Expression of Cathelicidin Antimicrobial Peptide (CAMP) in Obesity and During Weight Loss
}

\section{(요 $\odot \Theta$}

\author{
Authors \\ Alexandra Hochberg, Marissa Patz, Thomas Karrasch, Andreas Schäffler, Andreas Schmid
}

\begin{abstract}
Affiliation
Department of Internal Medicine III, University of Giessen, Giessen, Germany
\end{abstract}

Key words

Cathelicidin antimicrobial peptide, diet, bariatric surgery, adipose tissue, adipocyte

received 21.08.2020

accepted after revision $\quad 17.11 .2020$

published online 12.01 .2021

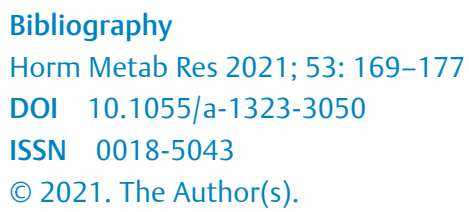

This is an open access article published by Thieme under the terms of the Creative Commons Attribution-NonDerivative-NonCommercial-License, permitting copying and reproduction so long as the original work is given appropriate credit. Contents may not be used for commecial purposes, or adapted, remixed, transformed or built upon. (https://creativecommons.org/ licenses/by-nc-nd/4.0/)

Georg Thieme Verlag KG, Rüdigerstraße 14, 70469

Stuttgart, Germany

Correspondence

Andreas Schmid PhD.

Department of Internal Medicine III

Giessen University Hospital

Klinikstraße 33

35392 Gießen

Germany

Tel.: + 4964199 30641, Fax.: + 496419930649

andreas.schmid@innere.med.uni-giessen.de

\section{ABSTRACT}

CAMP (Cathelicidin antimicrobial peptide) is synthesized and secreted by adipocytes and involved in adipose tissue (AT) innate immune response and host defense of subcutaneous AT against Gram positive bacteria. Data on the regulation of CAMP in obesity and during weight loss are scarce and reference values do not exist. Serum CAMP levels (ELISA) and AT gene expression levels (quantitative real time $P C R$ ) were investigated in two large and longitudinal (12 months) cohorts of severely obese patients undergoing either a low calorie diet (LCD; $n=79$ ) or bariatric surgery (BS; $n=156$ ). The impact of metabolic factors on CAMP expression in vitro was investigated in differentiated 3T3-L1 adipocytes. CAMP serum levels significantly increased after BS but not during LCD. Females had lower CAMP serum levels and lower gene expression levels in subcutaneous AT. CAMP was positively correlated to unfavorable metabolic factors/adipokines and negatively to favorable factors/adipokines. CAMP gene expression was higher in subcutaneous than in visceral AT but serum CAMP levels were not correlated to levels of AT gene expression. While certain bile acids upregulated CAMP expression in vitro, high glucose/insulin as well as GLP-1 had an inhibitory effect. There exist gender-specific and AT compartment-specific effects on the regulation of CAMP gene expression. Weight loss induced by BS (but not by LCD) upregulated CAMP serum levels suggesting the involvement of weight loss-independent mechanisms in CAMP regulation such as bile acids, incretins and metabolic factors. CAMP might represent an adipokine at the interface between metabolism and innate immune response.

\section{Introduction and Aims}

Antimicrobial peptides (AMPs) as part of the innate immune system are able to kill bacteria directly via membrane permeation and thus act somewhat like a molecular hole puncher [1]. However, AMPs have been increasingly recognized as pleiotropic and immunomodulating molecules regulating cell migration, wound healing, proliferation, differentiation, and angiogenesis. CAMP (Cathelicidin antimicrobial peptide, also named LL-37 or CAP18) represents the only human member of the Cathelicidin family and it is expressed in activated cells and organs such as epithelial cells, keratinocytes, neutrophils, monocytes, mast cells, skin, gastrointestinal and genito-urinary tract [1]. CAMP is composed of an N-terminal signal peptide, a conserved Cathelin-like domain, and a C-terminal antimicrobial domain that forms the mature antimicrobial peptide after cleavage by proteinase-3 [2]. CAMP has been regarded as a 
peptide of the innate immune system linking host defense and inflammation with angiogenesis and atherosclerosis [3].

Of note, Zhang et al. [4] reported for the first time in 2015 a fascinating link between CAMP and adipose tissue defense against Staphylococcus aureus (S. aureus). The authors demonstrated that proliferation and expansion of adipose tissue occurs after infection of subdermal adipose tissue by S. aureus[4]. Interestingly, this type of infection was more severe in murine models with impaired adipogenesis [4]. Inhibition of adipogenesis decreased CAMP expression and CAMP knockout mice had a decreased ability to fight against S. aureus growth [4]. Taken together, host defense against S. aureus was mediated by CAMP secreted from adipocytes [4]. A subsequent review article by Alcorn and Kolls commented on these results by using the term "killer fat"[5]. Gram positive bacteria such S. aureus and their respective lipopeptides are sensed and recognized by the toll-like receptors TLR-1/-2 and TLR-2/-6. Most recently, we could demonstrate that the TLR-2-CAMP-pathway is expressed and highly inducible in adipocytes [6]. During hormonally induced differentiation of murine 3T3-L1 pre-adipocytes into mature adipocytes, TLR-2 and CAMP were significantly upregulated in parallel. In mature adipocytes and also in pre-adipocytes, CAMP and TLR-2 expression were significantly increased upon stimulation with the TLR-1/-2 agonist Pam3Cys and the TLR-2/-6 agonist MALP-2, respectively [6, 7]. Taken together, CAMP is a differentiation-dependent secretory peptide of adipocytes fighting against adipose tissue infections. Thus, the regulation of CAMP in the context of obesity and during weight loss is of clinical interest.

It was our aim to

- quantify the serum levels of human CAMP in obese patients undergoing either low calorie diet (LCD) or bariatric surgery

(BS) before and after weight loss in two large and well-characterized clinical cohorts

- to investigate CAMP gene expression in subcutaneous and in visceral adipose tissue of patients undergoing bariatric surgery

- to correlate serum concentrations and gene expression levels with anthropometric and biochemical parameters

- to investigate the effects of metabolic factors and sex hormones on CAMP expression in vitro in adipocytes

\section{Material and Methods}

Serum samples and specimens from subcutaneous (abdominal) and visceral (intra-abdominal) adipose tissue were collected from the ROBS (Research in Obesity and Bariatric Surgery) study cohort. ROBS is an open-label, non-randomized, monocentric, prospective and observational (explorative and confirmatory) study of patients routinely undergoing either bariatric surgery (gastric sleeve or Roux-en-Y gastric bypass) or a low calorie formula diet (LCD) in the tertiary care center at the University of Giessen, Germany. The detailed information about this study cohort can be drawn from a recent publication [8]. Briefly, patients were treated by a multidisciplinary team of physicians and professionals from Internal Medicine, Endocrinology/Diabetology, Metabolic/Visceral Surgery, Psychosomatic Medicine/Psychotherapy, Nutritional Science/Dietetics, and Sports Medicine at the Obesity Centre at the University of Giessen, Germany. The study was approved by the local ethical committee at the University of Giessen, Germany (AZ 101/14). All patients gave informed consent and were informed about the aim of the study. Data anonymization and privacy policy were accurately applied. Obese patients with a BMI $>40 \mathrm{~kg} / \mathrm{m}^{2}$ or with a $\mathrm{BMI}>35 \mathrm{~kg} / \mathrm{m}^{2}$ and coexisting type 2 diabetes were consecutively admitted for bariatric surgery from January 2015 to February 2020. Exclusion criteria were: pregnancy, evidence of or suspicion on underlying endocrine diseases, untreated bulimia nervosa and binge eating behavior, use of illicit drugs, neoplasm, severe psychiatric disorders, psychosis, and psychopathologic instability.

\section{Serum measurement of CAMP concentrations}

The concentration of serum CAMP in each sample was measured in duplicates by ELISA (Hycultec $\mathrm{GmbH}$, Beutelsbach, Germany) and is expressed as mean \pm standard deviation. The intra- and inter-assay variation coefficient of the ELISA was $<20 \%$. The lower detection limit was $100 \mathrm{pg} / \mathrm{ml}$.

\section{Adipocyte cell culture and stimulation experiments}

Murine 3T3-L1-pre-adipocytes [9] were cultured at $37^{\circ} \mathrm{C}$ and $5 \%$ $\mathrm{CO}_{2}$ in DMEM (Dulbecco's Modified Eagle Medium, Biochrom AG, Berlin, Germany) that was supplemented with $10 \%$ newborn calf serum (NCS; Sigma-Aldrich, Deisenhofen, Germany) and $1 \%$ penicillin/streptomycin (PAN, Aidenbach, Germany). The cells were then differentiated into mature adipocytes at confluence by DMEM/ F12/glutamate-medium (Lonza, Basel, Switzerland) supplemented with $20 \mu \mathrm{M}$ 3-isobutylmethylxanthine (Serva, Heidelberg, Germany), $1 \mu \mathrm{M}$ corticosterone, $100 \mathrm{nM}$ insulin, $200 \mu \mathrm{M}$ ascorbate, $2 \mu \mathrm{g} / \mathrm{ml}$ apo-transferrin, $5 \%$ fetal calf serum (FCS), $1 \mu \mathrm{M}$ biotin, $17 \mu \mathrm{M}$ pantothenate (all from Sigma Aldrich, Deisenhofen, Germany), $1 \%$ penicillin/streptomycin, and $300 \mu \mathrm{g} / \mathrm{ml}$ Pedersen-fetuin (MP Biomedicals, Illkirch, France) $[10,11]$ for 9 days using a slightly modified protocol as reported in the literature [9, 12-15]. The cells were supplied with adipogenesis-inducing medium from day 0 until day 8 of differentiation, when cell culture medium was switched to serum-free conditions (with elevation of insulin supplementation to a concentration of $1 \mu \mathrm{M})$. At day 9 , mature adipocytes were adjusted to serum-free DMEM/F12/glutamate-medium lacking insulin for 3-5 h. With the start of stimulation experiments, cells were supplied with fresh serum-free medium. Cell phenotype was controlled by light-microscopy (appearance of extensive accumulation of lipid droplets). Mature adipocytes at day 9 of differentiation were used for stimulation experiments following overnight incubation under serum-free culture conditions. Mature adipocytes were cultured in serum-free media containing differing glucose ( 5.6 and $25 \mathrm{mM}$ ) and insulin doses $(0.2$ and $2 \mathrm{nM}$ ) for $18 \mathrm{~h}$. Further stimulation experiments were conducted with the sex hormones estradiol and testosterone ( 0.5 and $2 \mu \mathrm{M}$, each) and with GLP-1 (100 nM), all purchased by Sigma-Aldrich, Germany. Estradiol was dissolved in $\mathrm{H}_{2} \mathrm{O}$ and testosterone was dissolved in ethanol. GLP-1 was dissolved in $\mathrm{H}_{2} \mathrm{O}$. The following bile acids were tested and purchased from Sigma-Aldrich, Germany: tauromuricholic acid (TMCA; 1 and $10 \mu \mathrm{M})$, taurohyodeoxycholic acid (THDCA; $10 \mu \mathrm{M})$, cholic acid (CA; 10 and $100 \mu \mathrm{M})$, deoxycholic acid (DCA; 1 and $10 \mu \mathrm{M})$, and taurodeoxycholic acid (TDCA; 1 and $10 \mu \mathrm{M})$. Experiments were performed in mature adipocytes overnight (18 h) under serum-free culture conditions. LDH (lactate dehydrogenase) concentration was measured in the supernatants of all stimulation experiments 
(Cytotoxicity Detection Kit, Roche, Mannheim, Germany) in order to exclude any unexpected cytotoxic effects.

\section{Isolation of mRNA from human tissues}

Total mRNA was isolated from frozen human subcutaneous and visceral adipose tissue. Human subcutaneous and visceral adipose tissue was resected in obese patients undergoing bariatric surgery and participating in the ROBS study cohort [8]. Gene expression was quantified by reverse transcription of $300 \mathrm{ng}$ RNA (QuantiTect Reverse Transcription Kit from Qiagen, Hilden, Germany) and subsequent real-time PCR (RT-PCR) (iTaq Universal SYBR Green Supermix, CFX Connect RT-PCR system; Bio-Rad, Munich, Germany) of the corresponding CDNA as mentioned below in detail.

\section{Quantitative real-time PCR analysis of human and murine mRNA expression}

Gene expression of human CAMP during hormonally induced differentiation of 3T3-L1 pre-adipocytes as well as in murine and human adipose tissue depots was quantified by reverse transcription of isolated RNA and subsequent RT-PCR. The following primer sequences were used:

Murine Cathelicidin: 5'-GTGGCTGGCCTGGAGAAGAT-3'/5'-TT GTCATCTACGGGCACAAAG-3'

Human Cathelicidin: 5'-TAGATGGCATCAACCAGCGG-3'/5'-CTGGGTCCCCATCCATCGT-3'

Expression levels of the target gene were normalized to the gene expression of GAPDH as a house keeping gene as done by other experienced groups [16]. The primer-pairs used were: $5^{\prime}$-TGTCCGTCGTGGATCTGAC-3'/5'-AGGGAGATGCTCAGTGTTGG-3' (mouse), 5'-GAGTCCACTGGCGTCTTCAC-3'/5'-CCAGGGGTGCTAAGCAGTT-3' (human). All oligonucleotides used were purchased from Metabion (Martinsried, Germany).

\section{Data base and statistical analysis}

For explorative data analysis, a statistical software package (SPSS 22.0) was used. CAMP serum concentrations and gene expression levels did not follow a Gaussian distribution. Non-parametric numerical parameters were analyzed by the Mann-Whitney U-test (for 2 unrelated samples), the Kruskal-Wallis test ( $>2$ unrelated samples), the Wilcoxon test (for 2 related samples) or the Friedman test ( $>2$ related samples). Correlation analysis was performed by using the Spearman test. Partial correlation analysis was applied to control for possible covariates. A p-value below 0.05 (two tailed) was considered as statistically significant. In the figures, the bars are showing the mean values and the whiskers are giving the SEM (standard error of the mean).

\section{Results}

\section{Serum CAMP concentrations in severely obese patients undergoing either a low calorie formula diet (LCD) or bariatric surgery (BS)}

Since reference values of human serum CAMP concentrations have not yet been evaluated in the literature systematically, basal CAMP concentrations were measured in two large cohorts of patients undergoing either BS ( $n=156$; 122 females, 34 males; mean age: $40.1 \pm 11.0$ years; mean BMI: $53.6 \pm 6.6 \mathrm{~kg} / \mathrm{m}^{2}$; BMI range: $40.9-$ $\left.83.7 \mathrm{~kg} / \mathrm{m}^{2}\right)$ or LCD ( $\mathrm{n}=79 ; 51$ females, 28 males; mean age: $42.0+12.1$ years; mean BMI: $44.0 \pm 5.5 \mathrm{~kg} / \mathrm{m}^{2}$; BMI range: $31.9-$ $59.2 \mathrm{~kg} / \mathrm{m}^{2}$ ). The detailed study characteristics can be drawn from a recent publication [8]. - Table 1 summarizes mean values \pm SEM/ $S D$, median, range, variance and gender-specific concentrations of CAMP at a glance. The maximum range of CAMP over the two cohorts was $9.15-90.37 \mathrm{ng} / \mathrm{ml}$. Females had significantly lower basal CAMP concentrations when compared to males in both cohorts

- Table 1 Explorative data analysis of CAMP concentrations in human sera of patients undergoing either bariatric surgery (BS) or low calorie diet (LCD).

\begin{tabular}{|c|c|c|c|c|}
\hline \multirow[t]{2}{*}{ CAMP (ng/ml) } & \multicolumn{2}{|c|}{$\begin{array}{l}\text { Bariatric surgery (BS) }(n=156) \\
\text { Roux-en-Y gastric bypass } 121(77.6 \%) \\
\text { Sleeve gastrectomy } 35(22.4 \%)\end{array}$} & \multicolumn{2}{|c|}{ Low calorie diet $(L C D)(n=79)$} \\
\hline & Vo $(n=156)$ & V12 $(n=91)$ & V0 $(n=79)$ & V12 $(n=66)$ \\
\hline Mean \pm SEM & $39.48 \pm 1.10$ & $46.02 \pm 1.66$ & $42.35 \pm 3.64$ & $29.31 \pm 1.65$ \\
\hline SD & 13.76 & 15.81 & 32.37 & 13.42 \\
\hline Median & 37.25 & 42.70 & 30.44 & 26.40 \\
\hline Range & $14.35-91.04$ & $9.61-98.69$ & $10.91-190.37$ & $9.15-94.16$ \\
\hline Variance & 189.47 & 249.94 & 1047.55 & 180.04 \\
\hline Mean \pm SEM females & $38.25 \pm 1.18^{*}(n=122)$ & $44.39 \pm 1.76(n=69)$ & $37.62 \pm 4.55^{\circ}(n=51)$ & $28.49 \pm 1.61(n=46)$ \\
\hline Mean \pm SEM males & $43.91 \pm 2.68^{*}(n=34)$ & $51.15 \pm 3.94(n=22)$ & $50.97 \pm 5.84^{\circ}(n=28)$ & $31.20 \pm 4.05(n=20)$ \\
\hline
\end{tabular}



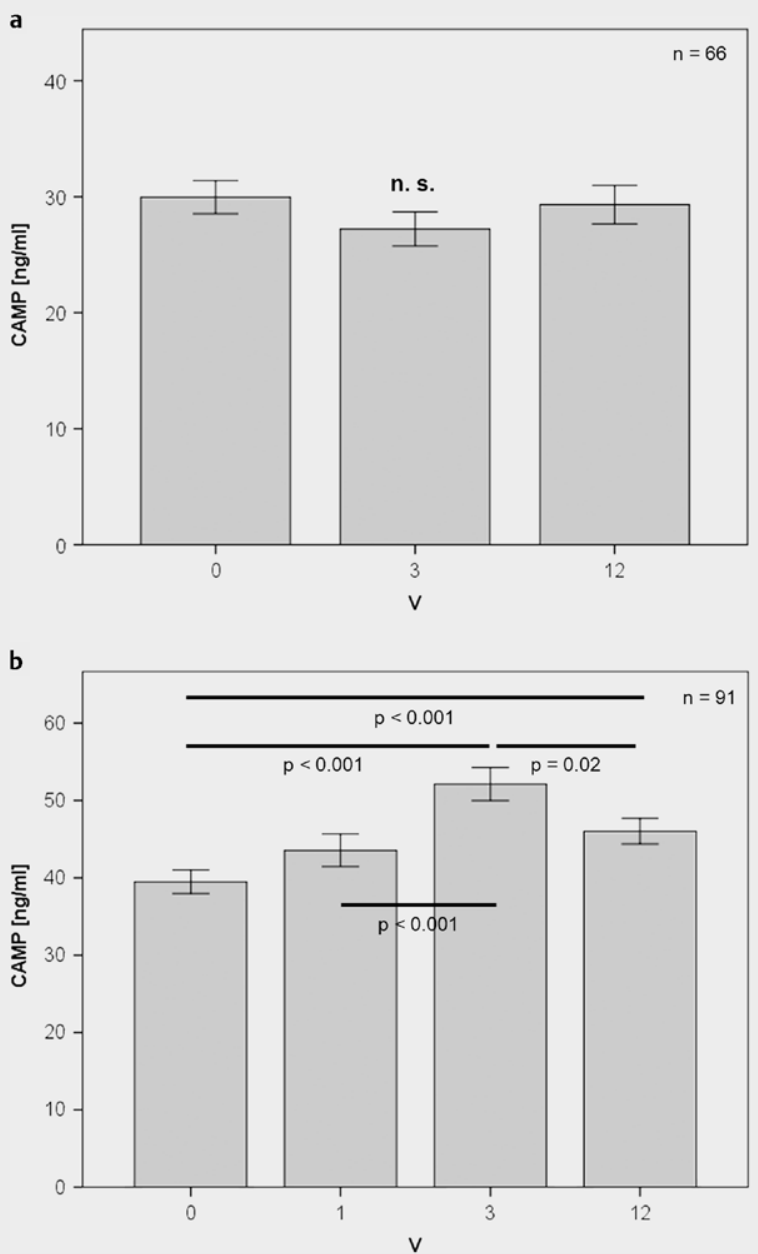

- Fig. 1 Serum CAMP concentrations during weight loss in patients undergoing low calorie diet (panel a) or bariatric surgery (panel b). Serum CAMP concentrations $(\mathrm{ng} / \mathrm{ml}$ ) were measured by ELISA over 12 months. CAMP: Cathelicidin antimicrobial peptide; n.s.: Not significant; V: Clinical visits (months). For statistical analysis, the Friedman test was used.

( ${ }^{*} \mathrm{p}=0.033$ and ${ }^{\circ} \mathrm{p}=0.006$, respectively). We could demonstrate that CAMP serum levels during weight loss were strongly influenced by the clinical approach of weight reduction. The time course of

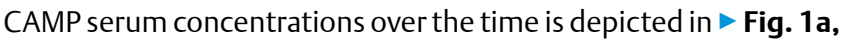
b. In patients during LCD ( $\mathbf{F i g}$. 1a), CAMP concentrations remained unchanged over the time. In contrast, CAMP levels increased significantly and in a stepwise manner $(p<0.001)$ from V0 to V3 ( $\triangleright$ Fig. 1b) after BS. Between V3 and V12, a significant decline occurred $(p=0.02)$ but levels at $V 12$ remained increased when compared to $\mathrm{V} 0$ pre-study levels $(p<0.001)$.

\section{Correlation analysis of CAMP serum concentrations with weight loss associated changes in anthropometric and clinical parameters}

We aimed to test whether changes in anthropometric and clinical parameters were correlated to basal CAMP levels as well as to changes of CAMP levels ( $\triangle$ CAMP) over the time. At first, basal CAMP levels were correlated with changes in anthropometric and clinical parameters upon weight loss after 12 months in the subgroups of BS and LCD ( $\triangleright$ Table 2, panel a). There was a positive correlation $(p=0.025$; rho $=+0.252)$ of $\Delta \mathrm{HbA}_{1 c}$ levels with basal CAMP concentrations in BS patients but not in LCD patients. Next, changes of CAMP concentrations ( $\triangle \mathrm{CAMP}$ ) over the time were correlated with changes in anthropometric and clinical parameters ( $>$ Table $\mathbf{2}$, panel b). There was a positive correlation of $\Delta$ CAMP with $\Delta$ body fat $(\%)$ in BS patients and with $\Delta$ body weight in LCD patients.

\section{Correlation analysis of CAMP mRNA expression in subcutaneous and visceral adipose tissue with anthropometric and biochemical parameters}

In our large cohort of patients undergoing BS $(n=156)$, we had the possibility to analyze mRNA expression quantitatively in subcutaneous and visceral adipose tissue. At first, basal CAMP serum levels prior to surgery were correlated with clinical parameters in all BS patients ( $\vee$ Table 3, panel a). Basal CAMP levels were positively correlated with resistin $(p=0.003$; rho $=+0.242)$ and triglycerides $(p=$ 0.005 ; rho $=+0.239)$, and negatively with $\mathrm{HDL}$ cholesterol $(p=0.038$; rho $=-0.176)$. In subcutaneous adipose tissue ( $>$ Table 3 , panel $\mathbf{b}$ ), CAMP expression was negatively correlated with adiponectin $(p=0.003 ;$ rho $=-0.24)$. In visceral adipose tissue ( $\triangleright$ Table 3, panel c), CAMP expression was positively correlated with $\mathrm{BMI}(\mathrm{p}=0.02$; $r h o=+0.186), H_{b A}(p=0.031 ;$ rho $=+0.185)$, and triglycerides $(p=0.026 ;$ rho $=+0.188)$. Taken together, CAMP serum levels and/ or CAMP expression in adipose tissue correlate positively with unfavorable metabolic factors (BMI, $\mathrm{HbA}_{1 c}$, triglycerides, resistin) and negatively with favorable factors (HDL cholesterol, adiponectin).

\section{CAMP gene expression is higher in subcutaneous adipose tissue when compared to visceral adipose tissue but not related to serum CAMP concentrations}

Since data on CAMP gene expression in human adipose tissue compartments are scarce, we analyzed gene expression by quantitative real time $P C R$ in subcutaneous and visceral adipose tissue of a large cohort of patients undergoing BS $(n=156)$. As depicted in $>$ Fig. $\mathbf{2 a}$, CAMP gene expression was significantly higher $(p<0.001)$ in subcutaneous adipose tissue ( about 20\%). Moreover, we aimed to investigate whether adipose tissue gene expression is directly correlated to the respective serum CAMP concentration in these pa-

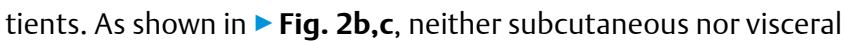
adipose tissue gene expression was directly correlated with serum CAMP levels.

\section{Females have lower CAMP gene expression levels than males in subcutaneous adipose tissue but not in visceral adipose tissue}

Since females are characterized by lower serum CAMP concentrations ( $\triangleright$ Table 1), we intended to clarify whether there exist gender-specific effects of gene expression in adipose tissue compartments. In contrast to visceral adipose tissue, females were characterized by significantly lower ( $p=0.01$ ) CAMP gene expression levels ( about $20 \%$ ) in subcutaneous adipose tissue ( $>$ Fig. 2 d). 


\section{Effect of glucose and insulin concentrations on CAMP expression in vitro in differentiated 3T3-L1 adipocytes}

Since glucose and insulin concentrations are crucial metabolic parameters in the context of obesity, we aimed to test their effects on CAMP expression in adipocytes in vitro under standardized conditions. - Fig. 3a summarizes the effects of varying glucose and insulin concentrations on CAMP expression in differentiated 3T3-L1 adipocytes. Supraphysiologic glucose concentrations reduced CAMP expression by a nonsignificant trend when compared to cells exposed to low/normal glucose concentrations. Insulin in the higher dose also reduced CAMP expression, although this reduction closely missed statistical significance. However, the combined exposure to high glucose and high insulin concentrations showed the most impressive effect regarding the suppression of CAMP expression ( $p=0.004)$ by about $50 \%$. These data might argue for a synergistic inhibitory effect of glucose and insulin on CAMP expression in adipocytes and for a potential impact of hyperglycemic hyperinsulinemia (as seen in type 2 diabetes and obesity) on the regulation of CAMP expression.

\section{Effects of bile acids and GLP-1 on the regulation of CAMP expression in differentiated adipocytes in vitro}

Since the increase of CAMP concentrations during weight loss could exclusively be documented after bariatric surgery ( $\bullet$ Fig. $\mathbf{1 b}$ ) but not under low calorie diet ( $\triangleright$ Fig. 1a), effects independent of weight loss but induced by the surgical procedure itself might account for this observation. In this context, it seems important to remember that an elevation of systemic bile acids has been regularly reported after bariatric surgery $[17,18]$. Thus, we aimed to test the hypothesis that bile acids might regulate CAMP expression directly in adipocytes in vitro. $>$ Fig. $\mathbf{3 b}$ summarizes the effects of different bile acids on CAMP expression in adipocytes. TMCA and THDCA significantly induced CAMP expression $(p<0.05$ and $p<0.01$, respectively) whereas CA, DCA, and TDCA had no effect. Thus, systemic bile acids might represent potential mediators standing behind the increasing CAMP levels after BS.

Incretin hormones such as GLP-1 and others are commonly increased after BS $[19,20]$. GLP-1 significantly $(p=0.007)$ reduced CAMP expression in adipocytes ( $\bullet$ Fig. $\mathbf{3 c}$ ) in vitro. Thus, it could be speculated that the decline of CAMP concentrations after BS be-

- Table 2 Correlation analysis of basal CAMP serum concentrations with weight loss associated changes in anthropometric and clinical parameters (a). Correlation analysis of weight loss associated changes in CAMP serum concentrations with anthropometric/clinical parameters (b).

\begin{tabular}{|c|c|c|c|c|c|c|}
\hline \multirow{2}{*}{$\begin{array}{l}\text { Correlations with CAMP levels } \\
\text { Parameter }\end{array}$} & \multicolumn{3}{|c|}{ Bariatric surgery (BS) $(n=91)$} & \multicolumn{3}{|c|}{ Low calorie diet (LCD) $(n=66)$} \\
\hline & $\mathbf{n}$ & rho & $\mathbf{p}$ & $\mathbf{n}$ & rho & $\mathbf{p}$ \\
\hline \multicolumn{7}{|l|}{ a: } \\
\hline$\Delta$ body weight $(\mathrm{kg})$ & 87 & +0.021 & 0.845 & 65 & -0.068 & 0.590 \\
\hline$\Delta \mathrm{BMI}\left(\mathrm{kg} / \mathrm{m}^{2}\right)$ & 87 & +0.100 & 0.356 & 65 & +0.008 & 0.947 \\
\hline$\Delta$ body fat (\%) & 80 & +0.111 & 0.327 & 64 & -0.054 & 0.675 \\
\hline$\triangle \mathrm{WHR}$ & 72 & +0.200 & 0.092 & 59 & +0.184 & 0.164 \\
\hline$\triangle \mathrm{HbA1c}(\%)$ & 79 & +0.252 & $0.025^{*}$ & 64 & +0.109 & 0.389 \\
\hline$\Delta$ Total cholesterol (mg/dl) & 81 & -0.084 & 0.458 & 64 & +0.173 & 0.173 \\
\hline$\Delta \mathrm{LDL}$ cholesterol (mg/dl) & 81 & +0.108 & 0.336 & 64 & +0.196 & 0.121 \\
\hline$\Delta \mathrm{HDL}$ cholesterol (mg/dl) & 81 & -0.029 & 0.796 & 64 & +0.064 & 0.617 \\
\hline$\Delta$ Triglycerides $(\mathrm{mg} / \mathrm{dl})$ & 81 & -0.032 & 0.778 & 64 & -0.049 & 0.703 \\
\hline$\Delta \mathrm{CRP}(\mathrm{mg} / \mathrm{dl})$ & 86 & +0.003 & 0.975 & 64 & +0.001 & 0.991 \\
\hline \multicolumn{7}{|l|}{ b: } \\
\hline$\Delta$ body weight (kg) & 87 & +0.027 & 0.801 & 65 & +0.256 & 0.039 \\
\hline$\Delta \mathrm{BMI}\left(\mathrm{kg} / \mathrm{m}^{2}\right)$ & 87 & -0.010 & 0.928 & 65 & +0.222 & 0.075 \\
\hline$\Delta$ body fat (\%) & 80 & +0.277 & $0.013^{*}$ & 64 & +0.232 & 0.065 \\
\hline$\triangle \mathrm{WHR}$ & 72 & +0.008 & 0.950 & 59 & +0.136 & 0.303 \\
\hline$\Delta \mathrm{HbA}_{1 \mathrm{c}}(\%)$ & 79 & -0.129 & 0.258 & 64 & +0.026 & 0.838 \\
\hline$\Delta$ Total cholesterol $(\mathrm{mg} / \mathrm{dl})$ & 81 & +0.081 & 0.473 & 64 & +0.114 & 0.371 \\
\hline$\Delta \mathrm{LDL}$ cholesterol $(\mathrm{mg} / \mathrm{dl})$ & 81 & +0.016 & 0.890 & 64 & +0.066 & 0.602 \\
\hline$\Delta \mathrm{HDL}$ cholesterol $(\mathrm{mg} / \mathrm{dl})$ & 81 & -0.113 & 0.314 & 64 & -0.071 & 0.580 \\
\hline$\Delta$ Triglycerides $(\mathrm{mg} / \mathrm{dl})$ & 81 & +0.155 & 0.168 & 64 & +0.109 & 0.392 \\
\hline$\Delta \mathrm{CRP}(\mathrm{mg} / \mathrm{dl})$ & 86 & +0.001 & 0.999 & 64 & +0.197 & 0.119 \\
\hline
\end{tabular}

Basal CAMP levels were correlated with changes in anthropometric and clinical parameters upon weight loss after 12 months in the subgroups of BS $(n=91)$ and $L C D(n=66)$ subgroups. Changes $(\Delta)$ between V0 and V12 were correlated with basal CAMP concentrations by using the Spearman test and significant correlations are indicated by ${ }^{*}(p<0.05)$. Changes in systemic CAMP levels ( $\triangle$ CAMP) as well as in anthropometric and clinical parameters upon weight loss after 12 months were correlated by using the Spearman test. Significant correlations are indicated by ${ }^{*}(p<0.05)$. 
- Table 3 Correlation analysis of basal CAMP serum concentrations (a), mRNA expression levels in subcutaneous (b), and visceral (c) adipose tissue with basal anthropometric and clinical parameters.

a:

\begin{tabular}{|l|l|l|l|}
\hline Serum CAMP & \multicolumn{3}{|l}{ Bariatric surgery (BS) } \\
$(\mathbf{n = 1 5 6})$ & $\mathbf{n}$ & rho & $\mathbf{p}$ \\
\hline Parameter & 156 & -0.085 & 0.292 \\
\hline Age & 156 & +0.145 & 0.071 \\
\hline Body weight & 156 & +0.025 & 0.754 \\
\hline BMI & 131 & -0.089 & 0.314 \\
\hline Body fat (\%) & 135 & +0.053 & 0.541 \\
\hline WHR & 136 & -0.100 & 0.245 \\
\hline HbA1c & 139 & +0.004 & 0.960 \\
\hline Total cholesterol & 139 & +0.041 & 0.631 \\
\hline LDL cholesterol & 139 & -0.176 & $0.038^{*}$ \\
\hline HDL cholesterol & 139 & +0.239 & $0.005^{*}$ \\
\hline Triglycerides & 155 & -0.059 & 0.462 \\
\hline CRP & 150 & -0.062 & 0.452 \\
\hline Adiponectin & 150 & -0.076 & 0.353 \\
\hline Leptin & 150 & +0.242 & 0.003 \\
\hline Resistin & & & \\
\hline
\end{tabular}

b:

\section{CAMP expression in subcutaneous AT}

Parameter

Age

Body weight

BMI

Body fat (\%)

WHR

$\mathrm{HbA}_{1 \mathrm{c}}$

Total cholesterol

LDL cholesterol

HDL cholesterol

Triglycerides

CRP

Adiponectin

Leptin

Resistin

c:

\section{CAMP expression in visceral AT}

\begin{tabular}{|l|l|l|l|}
\hline Parameter & $\mathbf{n}$ & rho & $\mathbf{P}$ \\
\hline Age & 156 & +0.015 & 0.855 \\
\hline Body weight & 156 & +0.110 & 0.173 \\
\hline BMI & 156 & +0.186 & $0.020^{*}$
\end{tabular}

Continued

c:

\begin{tabular}{|c|c|c|c|}
\hline \multirow{2}{*}{$\begin{array}{l}\text { CAMP expression in visceral AT } \\
\text { Parameter }\end{array}$} & \multicolumn{3}{|c|}{$\begin{array}{l}\text { Bariatric surgery (BS) } \\
(n=156)\end{array}$} \\
\hline & $n$ & rho & $\mathbf{p}$ \\
\hline Body fat (\%) & 131 & +0.014 & 0.876 \\
\hline WHR & 135 & -0.077 & 0.375 \\
\hline $\mathrm{HbA}_{1 \mathrm{c}}$ & 136 & +0.185 & $0.031^{*}$ \\
\hline Total cholesterol & 139 & -0.001 & 0.995 \\
\hline LDL cholesterol & 139 & -0.041 & 0.635 \\
\hline HDL cholesterol & 139 & -0.036 & 0.672 \\
\hline Triglycerides & 139 & +0.188 & $0.026^{*}$ \\
\hline CRP & 155 & +0.045 & 0.582 \\
\hline Adiponectin & 150 & -0.057 & 0.487 \\
\hline Leptin & 150 & 0 & 1.000 \\
\hline Resistin & 150 & -0.017 & 0.837 \\
\hline
\end{tabular}

CAMP serum levels and mRNA expression in subcutaneous and visceral adipose tissue were correlated with anthropometric and clinical parameters in BS patients $(n=156)$. Significant correlations are indicated by * $(p<0.05)$. The Spearman test was used. AT: Adipose tissue; BMI: Body mass index; CRP: C-reactive protein; WHR: Waist-hip ratio.

tween V3 and V12 ( $\vee$ Fig. 1b) might be regulated by incretin hormones such as GLP-1. Interestingly, former experiments using $100 \mathrm{nM}$ GLP-1 could demonstrate an inhibition of progranulin gene expression in adipocytes [21]. Progranulin also plays a role in adipose tissue innate immunity.

Since females were shown to have lower CAMP serum concentrations ( $\vee$ Table 1 ) and lower CAMP gene expression levels in subcutaneous adipose tissue ( $\vee$ Fig. $\mathbf{2 d}$ ), sex hormones might regulate the expression of CAMP in adipocytes. Thus, differentiated 3T3-L1 adipocytes were incubated under increasing doses of testosterone and estradiol. However, neither testosterone nor estradiol were able to modulate CAMP expression significantly ( $\triangleright$ Fig. 3d). Thus, other (systemic) mechanisms seem to be responsible for gender-specific effects on CAMP expression.

\section{Discussion}

The adipocytic innate immune system seems to orchestrate the defense of local (subcutaneous) adipose tissue compartments against Gram positive bacteria such as Staphylococci $[4,5]$. This mechanism might represent an evolutionarily conserved machinery that is highly reasonable and meaningful since adipocytes and adipose tissue often reside at tissue interfaces involved in complicated local infections such as bowel perforation (mesenteric adipose tissue), acute pancreatitis (retroperitoneal adipose tissue), chronic inflammatory bowel disease (mesenteric adipose tissue), arthritis (periarticular adipose tissue), ophthalmitis (retro-orbital adipose tissue), soft tissue infection (subcutaneous adipose tissue), 


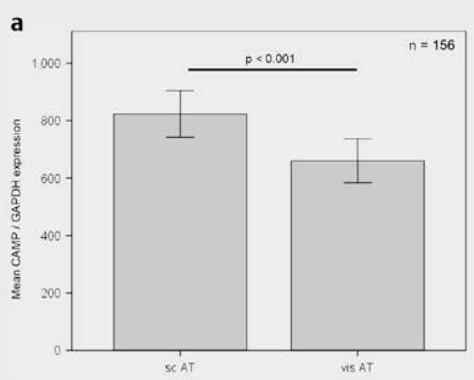

b
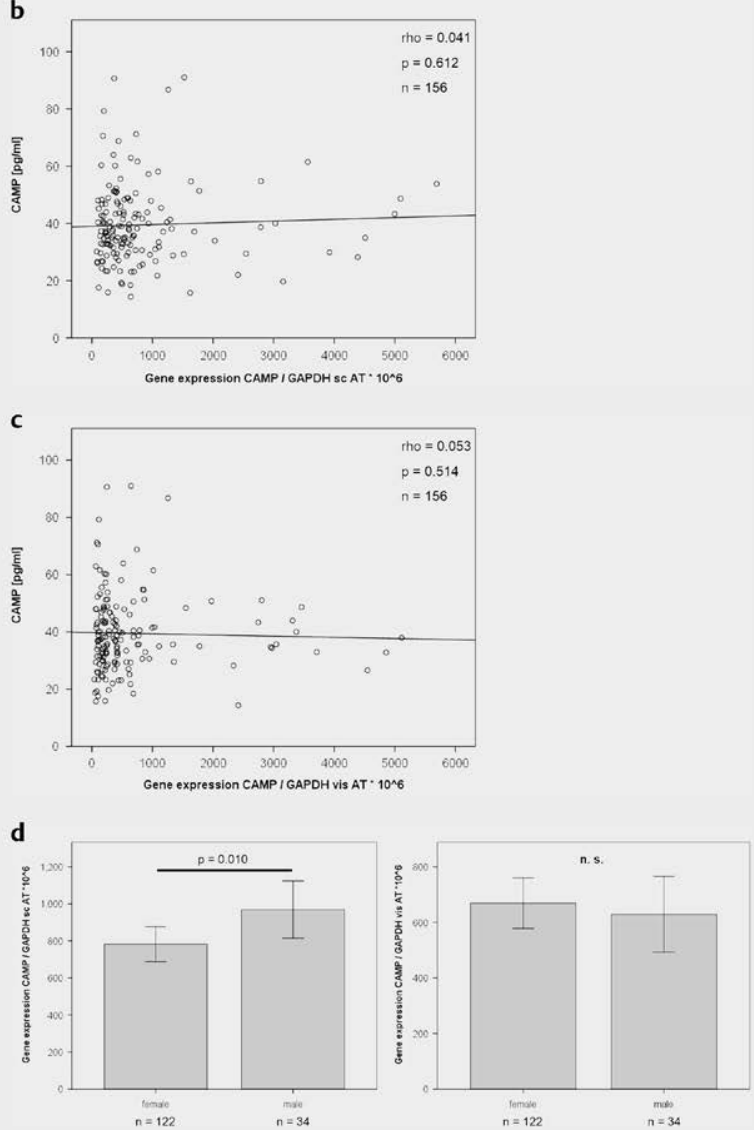

Fig. 2 Gene expression analysis of CAMP in adipose tissue compartments. The mRNA expression of CAMP was quantified by real time PCR (in relation to GAPDH) in subcutaneous (sc) and visceral (vis) adipose tissue (AT) of $n=156$ patients undergoing bariatric surgery (BS). In parallel, serum CAMP concentrations were measured by ELISA in these patients prior to surgery. GAPDH, glyceraldehyde-3-phosphate dehydrogenase. Panel a: CAMP gene expression is higher in subcutaneous than in visceral adipose tissue $(p<0.001$; Wilcoxon test). Panel b: Subcutaneous adipose tissue CAMP expression is not a predictor of serum CAMP concentrations (Spearman test). Panel c: Visceral adipose tissue CAMP expression is not a predictor of serum CAMP concentrations (Spearman test). Panel d: Gender-specific analysis of CAMP gene expression in adipose tissue compartments. In contrast to visceral adipose tissue, CAMP gene expression is lower in females when compared to males $(p=0.01)$ in subcutaneous adipose tissue (Mann-Whitney U test). and many others [22]. Thus, detailed knowledge of the physiological and pathophysiological regulation of CAMP is important and of translational impact. The clinical perspective [5] derived from the data published by Zhang et al. [4] raises the possibility that adipocytes use CAMP in order to fight against Gram positive bacterial infection of adipose tissue. Soft tissue infections in obesity are a common clinical problem involving subcutaneous adipose tissue.

In our experiments in vitro, high glucose plus high insulin concentrations resemble the metabolic situation of poorly controlled type 2 diabetes mellitus and/or obesity (hyperglycemic hyperinsulinemia). We could demonstrate that glucose and insulin synergistically decrease adipocytic CAMP expression. Based on these observations, one could speculate that high rates of soft tissue infections in obesity and/or diabetes mellitus type 2 might be worsened by decreased CAMP expression in local subcutaneous adipose tissue. Future translational and clinical studies should be performed in order to test the suitability of CAMP as a diagnostic marker of soft tissue infection and treatment. Moreover, CAMP might has the potential as an endogenous and inducible anti-microbial molecule (drug target).

We could demonstrate that serum CAMP levels increase significantly after BS but not during LCD. Thus, mechanisms other than weight reduction seem to have caused this effect. Bile acids have been reported to increase regularly and rapidly after BS such as RYGB $[17,18]$. Moreover, bile acids have recently been recognized as pleiotropic hormones affecting white adipose tissue function and adipokine secretion $[23,24]$. Thus, we investigated a broad panel of human bile acid species in murine adipocytes for their potential to modulate CAMP gene expression. We could demonstrate that certain bile acids in a non-toxic dose range are able to upregulate CAMP expression. This observation might be explained by the fact that adipocytes synthesize one of the main bile acid-responsive transcription factor, FXRalpha (farnesoid-X-receptor-alpha). Tauromurocholic acid (TMCA) induced CAMP expression in the present study. It is important to remember that 3T3-L1 adipocytes represent a murine cell line and that TMCA is one of the main bile acids found in mice. TMCA differs from primary bile acids found in humans such as cholic acid (CA) which had no effect in our experiments. The secondary and conjugated bile acids deoxycholic acid (DCA) and taurodeoxycholic acid (TDCA) were also without any effects on CAMP expression. Moreover, the results are supported by our recent study on white adipocytes providing evidence of a functional bile acid signaling pathway [24] in adipocytes. Thus, bile acids might represent future and therapeutic modulators of CAMP in the context of infection and inflammation similar to certain bile acids such as ursodeoxycholic acid and obeticholic acid that have been used for the treatment of inflammatory liver diseases.

Since CAMP represents an anti-microbial peptide, acute infections might upregulate CAMP serum levels. However, patients were investigated by a physician at every time point of the study and we excluded patients with signs or symptoms of a clinical infection. Moreover (as already published earlier [8]), CRP concentrations were evaluated and shown not to differ between bariatric patients and patients under diet.

Incretin hormones such as GLP-1 have also been reported to increase regularly after BS $[19,20]$. Since we observed a decline of elevated CAMP levels between V3 and V12 after BS, we tested 
a

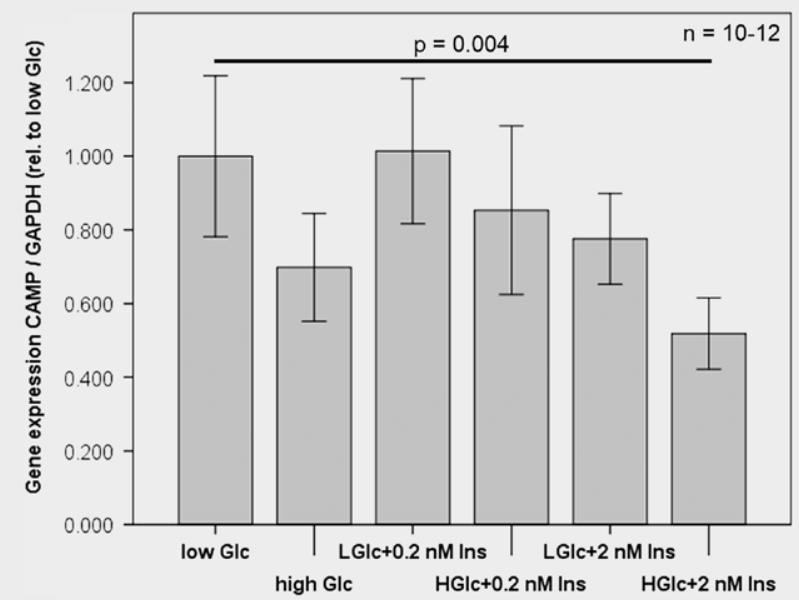

b

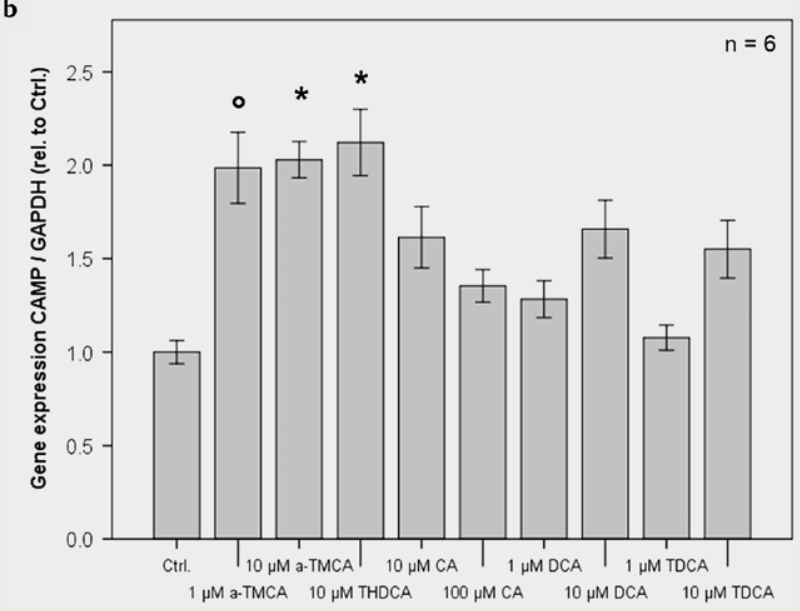

c

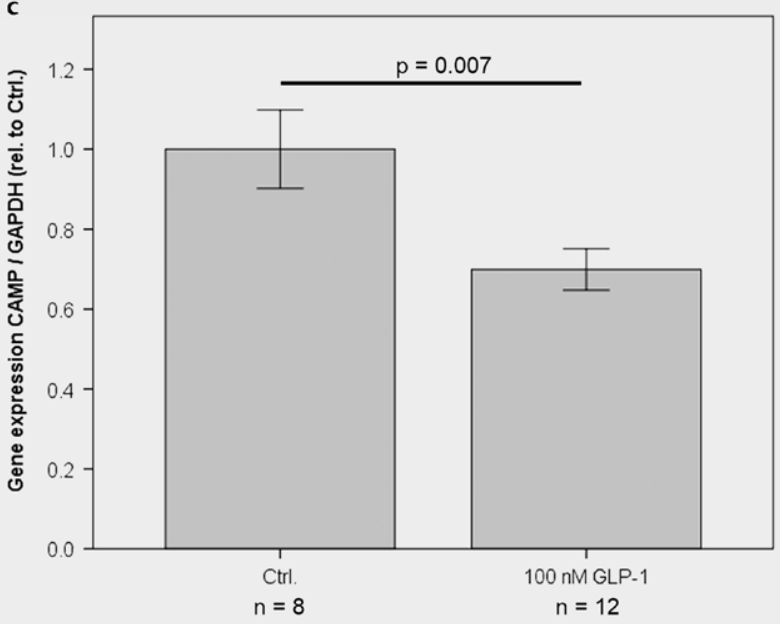

d

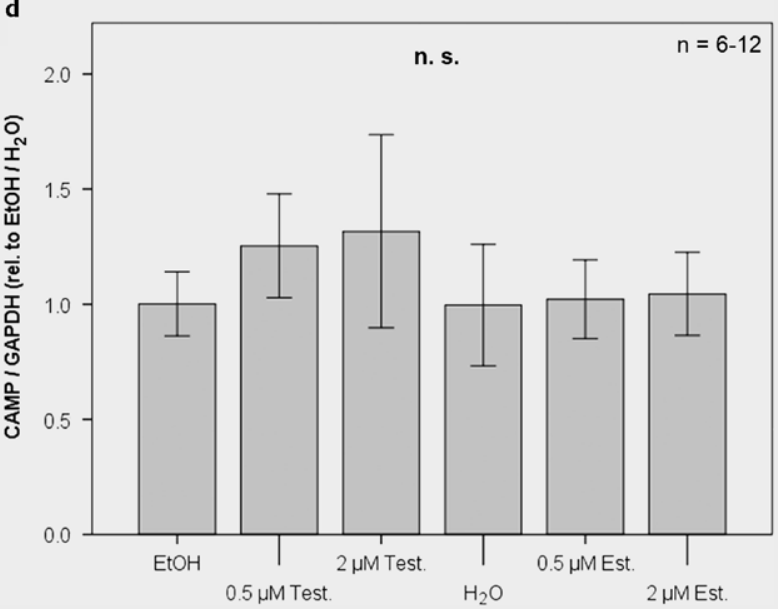

- Fig. 3 Regulation of CAMP gene expression in vitro in differentiated 3T3-L1 adipocytes. CAMP mRNA was measured by quantitative real-time PCR. CAMP, Cathelicidin anti-microbial peptide; GAPDH, glyceraldehyde-3-phosphatedehydrogenase. Panel a: High concentrations of glucose and insulin synergistically suppress the gene expression of CAMP in adipocytes in vitro. Differentiated 3T3-L1 adipocytes were incubated under varying glucose and insulin concentrations. Cells were exposed to low/normal (low Glc/LGlc) glucose concentrations (5.6 mM) and to supraphysiologic (high $\mathrm{Glc} / \mathrm{HGlc}$ ) glucose concentrations $(25 \mathrm{mM})$ alone or in combination with low $(0.2 \mathrm{nM})$ and high $(2.0 \mathrm{nM})$ insulin (Ins) concentrations for $18 \mathrm{~h}$. $\mathrm{n}=10-$ 12 wells were investigated per experimental setting. The combined exposure to high glucose and high insulin concentrations significantly reduced CAMP gene expression ( $p=0.004$; Kruskal-Wallis test). Panel $\mathbf{b}$ : Effects of bile acids on CAMP expression in adipocytes in vitro. Differentiated 3T3-L1 adipocytes were exposed to a broad panel of bile acids. Tauromuricholic acid (TMCA; 1 and 10 $\mu$ M), taurohyodeoxycholic acid (THDCA; $10 \mu M$ ), cholic acid $(C A ; 10$ and $100 \mu \mathrm{M})$, deoxycholic acid (DCA; 1 and $10 \mu \mathrm{M})$, and taurodeoxycholic acid (TDCA; 1 and $10 \mu \mathrm{M}$ ) were investigated. ${ }^{\circ} \mathrm{P}<0.05 ;{ }^{*} \mathrm{P}<0.01$

(Kruskal-Wallis test). $n=6$ wells were investigated per experimental setting. Panel $\mathbf{c}$ : Effect of GLP-1 on CAMP expression in adipocytes in vitro. GLP-1 significantly $(p=0.007)$ inhibited CAMP gene expression (Kruskal-Wallis test). GLP-1: Glucagon-like peptide-1; Ctrl.: Control. $n=8-12$ wells were investigated per experimental setting. Panel d: Effects of testosterone and estradiol on CAMP expression in adipocytes in vitro. Neither testosterone nor estradiol significantly (Kruskal-Wallis test) influenced CAMP expression. Test.: Testosterone; Est.: Estradiol; $n=6-12$ wells were investigated per experimental setting.

GLP-1 for its ability to suppress CAMP expression in adipocytes in vitro. This hypothesis could be verified by a large number of cell culture experiments in vitro. However, it is a limitation of the present study that bile acid species and incretin hormones could not be measured in the two cohorts. Future studies have to address this question by using tandem mass spectrometry (LC-MS/MS) in order to correlate the bile acid metabolome [25] in obesity and after BS with CAMP levels. Moreover, recent data already proved [25] that the human bile acid metabolome is rapidly responsive to an oral lipid uptake. Thus, the modulation of bile acid metabolome (and also incretins) during LCD should be investigated systematically by LC-MS/MS.

The gender-specific effect showing reduced serum CAMP levels and reduced subcutaneous adipose tissue gene expression levels in females is interesting but remains unclear. Since sex hormones did not influence CAMP expression in adipocytes in vitro under serum-free conditions (and in the absence of incretins or bile acids), other systemic or multifactorial mechanisms seem to play a role. Moreover, adipose tissue is not the only potential cellular source of circulating CAMP. This is highlighted by the fact that neither subcutaneous nor visceral adipose tissue CAMP expression was corre- 
lated to the respective serum CAMP concentrations in 156 patients. Thus, other cellular sources of CAMP seem to exist. Based on this observation, CAMP cannot longer be regarded as a typical adipokine (such as adiponectin) with an adipocyte-specific expression and secretion profile. This has to be borne in mind when interpreting serum CAMP levels in future studies. The present study investigated CAMP mRNA expression in human total subcutaneous and visceral adipose tissue. The limitation is given by the fact that other cell types possibly expressing CAMP are present in total adipose tissue (stroma vascular cell fraction, immune cells). Since a reliable and well established human adipocyte cell line (without any syndromal or tumorigenic background) is lacking, future studies are necessary to collect human adipose tissue for the preparation of (pre-adipocyte) precursor cells and subsequent hormonal differentiation into mature adipocytes.

LPS as a potent TLR-4 ligand upregulates CAMP expression in neutrophils [26] and the effects of MALP-2 on CAMP expression in pre-adipocytes and in mature adipocytes was documented in two recent studies [6, 7]. Based on this, CAMP levels seem to be correlated with inflammatory response syndromes. This is important to remember because CAMP serum levels and/or CAMP expression in adipose tissue were shown to correlate positively with unfavorable metabolic factors (BMI, $\mathrm{HbA}_{1 \mathrm{c}}$, triglycerides, resistin) and negatively with favorable factors (HDL cholesterol, adiponectin) in the present study. To the best of our knowledge, the present study provides the largest cohort of obese patients that has been investigated in order to quantify circulating CAMP before and after weight loss. Thus, the present data might be helpful for the future establishment of reference values.

\section{Conclusions}

There exist gender-specific and AT compartment-specific effects on the regulation of CAMP gene expression. Weight loss induced by BS (but not by LCD) upregulated CAMP serum levels suggesting the involvement of weight loss-independent mechanisms on CAMP regulation such as bile acids, incretins, and metabolic factors. CAMP might represent an adipokine at the interface between metabolism and innate immune response. The presented serum concentrations in two large cohorts might be useful to establish reference values for human CAMP.

\section{Conflict of Interest}

The authors declare that they have no conflict of interest.

\section{References}

[1] Lee EY, Lee MW, Wong GCL. Modulation of toll-like receptor signaling by antimicrobial peptides. Semin Cell Dev Biol 2019; 88: 178-184

[2] Sorensen OE, Follin P, Johnsen AH et al. Human cathelicidin, hCAP-18, is processed to the antimicrobial peptide LL-37 by extracellular cleavage with proteinase 3. Blood 2001; 97: 3951-3959

[3] Koczulla R, von Degenfeld G, Kupatt $C$ et al. An angiogenic role for the human peptide antibiotic LL-37/hCAP-18. J Clin Invest 2003; 111: $1665-1672$
[4] Zhang L], Guerrero-Juarez CF, Hata T et al. Innate immunity. Dermal adipocytes protect against invasive Staphylococcus aureus skin infection. Science 2015; 347: 67-71

[5] Alcorn JF, Kolls JK. Physiology. Killer fat. Science 2015; 347: 26-27

[6] Schmid A, Karrasch T, Thomalla M et al. Innate immunity of adipose tissue in rodent models of local and systemic staphylococcus aureus infection. Mediators Inflamm 2017; 2017: 5315602

[7] Liggins MC, Li F, Zhang LJ et al. Retinoids Enhance the expression of cathelicidin antimicrobial peptide during reactive dermal adipogenesis. J Immunol 2019; 203: 1589-1597

[8] Brock J, Schmid A, Karrasch T et al. Progranulin serum levels and gene expression in subcutaneous vs visceral adipose tissue of severely obese patients undergoing bariatric surgery. Clin Endocrinol (Oxf) 2019; 91: 400-410

[9] Green H, Kehinde O. An established preadipose cell line and its differentiation in culture. II. Factors affecting the adipose conversion. Cell 1975; 5: 19-27

[10] Zaitsu H, Serrero G. Pedersen fetuin contains three adipogenic factors with distinct biochemical characteristics. J Cell Physiol 1990; 144: 485-491

[11] Bachmeier M, Loffler G. Adipogenic activities in commercial preparations of fetuin. Horm Metab Res 1994; 26: 92-96

[12] Green H, Kehinde O. Formation of normally differentiated subcutaneous fat pads by an established preadipose cell line. J Cell Physiol 1979; 101: 169-171

[13] Green H, Meuth M. An established pre-adipose cell line and its differentiation in culture. Cell 1974; 3: 127-133

[14] Cornelius P, MacDougald OA, Lane MD. Regulation of adipocyte development. Annu Rev Nutr 1994; 14: 99-129

[15] MacDougald OA, Lane MD. Transcriptional regulation of gene expression during adipocyte differentiation. Annu Rev Biochem 1995; 64: 345-373

[16] Singh P, Zhang $Y$, Sharma $P$ et al. Statins decrease leptin expression in human white adipocytes. Physiol Rep 2018; 6: 13566

[17] Penney NC, Kinross J, Newton RC et al. The role of bile acids in reducing the metabolic complications of obesity after bariatric surgery: a systematic review. Int J Obes 2015; 39: 1565-1574

[18] Noel OF, Still CD, Argyropoulos G et al. Bile acids, fxr, and metabolic effects of bariatric surgery. J Obes 2016; 2016: 4390254

[19] Ionut V, Burch M, Youdim A et al. Gastrointestinal hormones and bariatric surgery-induced weight loss. Obesity 2013; 21: 1093-1103

[20] Argyropoulos G. Bariatric surgery: prevalence, predictors, and mechanisms of diabetes remission. Curr Diabetes Rep 2015; 15: 15

[21] Schmid A, Hochberg A, Kreiss AF et al. Role of progranulin in adipose tissue innate immunity. Cytokine 2020; 125: 154796

[22] Schaffler A, Muller-Ladner U, Scholmerich J et al. Role of adipose tissue as an inflammatory organ in human diseases. Endocr Rev 2006; 27 : 449-467

[23] Bargut TCL, Souza-Mello V, Aguila MB et al. Browning of white adipose tissue: lessons from experimental models. Horm Mol Biol Clinical Invest 2017, 31:hmbci.2017.31.issue-1/hmbci-2016-0051/ hmbci-2016-0051.xml. doi: 10.1515/hmbci-2016-0051

[24] Schmid A, Schlegel J, Thomalla $M$ et al. Evidence of functional bile acid signaling pathways in adipocytes. Mol Cell Endocrinol 2019; 483: 1-10

[25] Schmid A, Neumann H, Karrasch T et al. Bile acid metabolome after an oral lipid tolerance test by Liquid Chromatography-Tandem Mass Spectrometry (LC-MS/MS). PLoS One 2016; 11: e0148869

[26] Wang TT, Nestel FP, Bourdeau V et al. Cutting edge: 1,25-dihydroxyvitamin D3 is a direct inducer of antimicrobial peptide gene expression. J Immunol 2004; 173: 2909-2912 